\title{
Thermodynamics and Luminosities of Rainbow Black Holes
}

\author{
Benrong $\mathrm{Mu}^{a, b} *$ Peng Wang ${ }^{b}+\dagger$ and Haitang Yang ${ }^{b, t} \ddagger$ \\ ${ }^{a}$ Physics Teaching and Research Section, College of Medical Technology, \\ Chengdu University of Traditional Chinese Medicine, Chengdu 611137, China \\ ${ }^{b}$ Center for Theoretical Physics, College of Physical Science and Technology, \\ Sichuan University, Chengdu, 610064, China and \\ ${ }^{c}$ Kavli Institute for Theoretical Physics China (KITPC), \\ Chinese Academy of Sciences, Beijing 100080, China
}

\begin{abstract}
Doubly special relativity (DSR) is an effective model for encoding quantum gravity in flat spacetime. As a result of the nonlinearity of the Lorentz transformation, the energy-momentum dispersion relation is modified. One simple way to import DSR to curved spacetime is "Gravity's rainbow", where the spacetime background felt by a test particle would depend on its energy. Focusing on the "Amelino-Camelia dispersion relation" which is $E^{2}=m^{2}+p^{2}\left[1-\eta\left(E / m_{p}\right)^{n}\right]$ with $n>0$, we investigate the thermodynamical properties of a Schwarzschild black hole and a static uncharged black string for all possible values of $\eta$ and $n$ in the framework of rainbow gravity. It shows that there are non-vanishing minimum masses for these two black holes in the cases with $\eta<0$ and $n \geq 2$. Considering effects of rainbow gravity on both the Hawking temperature and radius of the event horizon, we use the geometric optics approximation to compute luminosities of a 2D black hole, a Schwarzschild one and a static uncharged black string. It is found that the luminosities can be significantly suppressed or boosted depending on the values of $\eta$ and $n$.
\end{abstract}

\footnotetext{
*Electronic address: mubenrong@uestc.edu.cn

${ }^{\dagger}$ Electronic address: pengw@scu.edu.cn

$\ddagger$ Electronic address: hyanga@scu.edu.cn
} 


\section{Contents}

I. Introduction

II. Hamilton-Jacobi Method

III. Thermodynamics of Black Holes in Rainbow Gravity

B. Static Uncharged Black String

IV. Luminosities of Black Holes in Rainbow Gravity

A. 2D Black Hole

B. 4D Spherically Symmetric Black Hole

C. 4D Cylindrically Symmetric Black Hole

\section{Acknowledgments}

References

\section{INTRODUCTION}

The Hawking radiation was a remarkable prediction of quantum field theory in curved spacetime. As Stephen Hawking demonstrated[1], a Schwarzschild black hole emits radiation just like an ordinary blackbody at temperature $T=\hbar \kappa / 2 k_{B} \pi$, where $\kappa$ is the surface gravity. Soon after this discovery, it was realized that there might be the "trans-Planckian" problem[2]. It appears that the Hawking radiation originates from the modes with huge initial frequencies, well beyond the Planck mass $m_{p}$, which undergo exponential high gravitational red-shifting near the horizon. As a result, the Hawking's prediction relies on the validity of quantum field theory in curved spacetime to arbitrary high energies. On the other hand, quantum field theory is considered more like an effective field theory of an underlying theory whose nature remains unknown. This observation poses the question of whether any unknown physics at the Planck scale could strongly influence the Hawking radiation. 
Although a complete understanding of the trans-Planckian problem requires a full theory of quantum gravity, there are various attempts using effective models to address this problem. Among them is Doubly Special Relativity (DSR) [3 6], where the transformation laws of special relativity are modified at very high energies. In DSR, the energy-momentum dispersion relation for a massive particle of mass $m$ is modified to

$$
E^{2} f^{2}\left(E / m_{p}\right)-p^{2} g^{2}\left(E / m_{p}\right)=m^{2},
$$

where $m_{p}$ is the Planck mass, and $f(x)$ and $g(x)$ are two unknown functions with the following properties

$$
\lim _{x \rightarrow 0} f(x)=1 \text { and } \lim _{x \rightarrow 0} g(x)=1 .
$$

It has been shown that the modified dispersion relation (MDR) might play a role in astronomical and cosmological observations, such as the threshold anomalies of ultra high energy cosmic rays and $\mathrm{TeV}$ photons [7-12]. Moreover, thermodynamics of black holes have been explored in the framework of the MDR[13-18].

One of the most popular choice for the functions $f(x)$ and $g(x)$ has been proposed by Amelino-Camelia et al.[19, 20], which gives

$$
f(x)=1 \text { and } g(x)=\sqrt{1-\eta x^{n}} .
$$

Usually one has $n>0$. As shown in [20], this formula is compatible with some of the results obtained in the Loop-Quantum-Gravity approach and reflects the results obtained in $\kappa$-Minkowski and other noncommutative spacetimes. Phenomenological implications of this "Amelino-Camelia (AC) dispersion relation" are also reviewed in [20].

Nevertheless, the non-linear realization of the Lorentz transformation in the framework of DSR results in a very complicated definition of the dual position space. To circumvent this difficulty, Magueijo and Smolin[21] proposed the "Gravity's rainbow", where the spacetime background felt by a test particle would depend on its energy. Consequently, the energy of the test particle deforms the background geometry and hence the dispersion relation. As regards the metric, it would be replaced by a one parameter family of metrics which depends on the energy of the test particle, forming a "rainbow metric". Specifically, for the Schwarzschild solution, the corresponding "rainbow metric" is

$$
d s^{2}=\left(1-\frac{2 G M}{r}\right) \frac{d t^{2}}{f^{2}\left(E / m_{p}\right)}-\frac{1}{g^{2}\left(E / m_{p}\right)}\left[\frac{d r^{2}}{1-\frac{2 G M}{r}}+r^{2}\left(d \theta^{2}+\sin ^{2} \theta d \phi^{2}\right)\right],
$$


which is the spherically symmetric solution to the distorted Einstein's Field equations given in [21]. Note that $E$ is the energy of the probing particle. To obtain the modified Schwarzschild metric from the usual one, it appears from eqn. (4) that we can simply make replacements $d t \rightarrow d t / f\left(E / m_{p}\right)$ for the time coordinate and $d x^{i} \rightarrow d x^{i} / g\left(E / m_{p}\right)$ for all spatial coordinates. In fact, such procedure also works for the black objects besides the Schwarzschild metric [22]. The rainbow gravity formalism has received a lot of attentions recently, for instance, in cosmology [23-26] and black hole physics [27 34$]$.

In this paper, we will study thermodynamics and luminosities of black holes in the framework of rainbow gravity. The remainder of our paper is organized as follows. In section III, the deformed Hamilton-Jacobi equations for scalars, spin 1/2 fermions and vector bosons are derived in the framework of rainbow gravity. We then solve the Hamilton-Jacobi equations to obtain tunneling rates. The temperatures and entropies of a rainbow Schwarzschild black hole and a rainbow static uncharged black string is computed in section III. In section IV, we calculate the luminosities of a $2 \mathrm{D}$ rainbow black hole, a 4D rainbow spherically symmetric one, and a 4D rainbow cylindrically symmetric one. Section $\mathrm{V}$ is devoted to our conclusion. Throughout the paper we take Geometrized units $c=G=1$, where the Planck constant $\hbar$ is square of the Planck mass $m_{p}$.

\section{HAMILTON-JACOBI METHOD}

After the Hawking's original derivation, there have been some other methods proposed to understand the Hawking radiation. Recently, a semiclassical method of modeling Hawking radiation as a tunneling process has been developed and attracted a lot of attention. This method was first proposed by Kraus and Wilczek [35, 36], which is known as the null geodesic method. They employed the dynamical geometry approach to calculate the imaginary part of the action for the tunneling process of s-wave emission across the horizon and related it to the Hawking temperature. Later, the tunneling behaviors of particles were investigated using the Hamilton-Jacobi method[37-39]. In the Hamilton-Jacobi method, one ignores the self-gravitation of emitted particles and assumes that its action satisfies the relativistic Hamilton-Jacobi equation. The tunneling probability for the classically forbidden trajectory from inside to outside the horizon is obtained by using the Hamilton-Jacobi equation to calculate the imaginary part of the action for the tunneling process. 
In this section, the Hamilton-Jacobi equations for scalars, spin $1 / 2$ fermions and vector bosons in the rainbow metric are derived. The particles' tunneling rates across the event horizon $r=r_{h}$ of the rainbow metric (6) are then computed by solving the Hamilton-Jacobi equations. We here consider a static black hole with the line element

$$
d s^{2}=B(r) d t^{2}-\frac{d r^{2}}{B(r)}-C\left(r^{2}\right) h_{a b}(x) d x^{a} d x^{b},
$$

whose rainbow metric is obtained by $d t \rightarrow d t / f\left(E / m_{p}\right), d r \rightarrow d r / g\left(E / m_{p}\right)$, and $d x_{i} \rightarrow$ $d x_{i} / g\left(E / m_{p}\right)$

$$
d s^{2}=\frac{B(r) d t^{2}}{f^{2}\left(E / m_{p}\right)}-\frac{d r^{2}}{g^{2}\left(E / m_{p}\right) B(r)}-\frac{C\left(r^{2}\right) h_{a b}(x) d x^{a} d x^{b}}{g^{2}\left(E / m_{p}\right)} .
$$

The function $B(r)$ has a simple zero at $r=r_{h}$ with $B^{\prime}\left(r_{h}\right)$ being finite and nonzero. The vanishing of $B(r)$ at point $r=r_{h}$ indicates the presence of an event horizon. For simplicity, we assume that the particles are massless.

In the rainbow metric $d s^{2}=\tilde{g}_{\mu \nu}(E) d x^{\mu} d x^{\nu}$, the massless scalar field $\phi$ obeys the KleinGordon equation

$$
\tilde{\nabla}^{\mu} \tilde{\nabla}_{\mu} \phi=0
$$

where $\tilde{\nabla}_{\mu}$ is the covariant derivative associated with $\tilde{g}_{\mu \nu}(E)$ and the index $\mu$ is lowered or raised by $\tilde{g}_{\mu \nu}(E)$. Making the ansatz for $\phi$ which is

$$
\phi=\exp \left(\frac{i I}{\hbar}\right),
$$

substituting it into eqn. (7), and expanding eqn. (7) in powers of $\hbar$, the leading order gives the Hamilton-Jacobi equation for a massless scalar particle

$$
\tilde{g}_{\mu \nu}(E) \partial^{\mu} I \partial^{\nu} I=0 .
$$

The Dirac equation for a spin- $1 / 2$ fermion field $\psi$ takes the form of

$$
i \tilde{\gamma}_{\mu}\left(\partial^{\mu}+\tilde{\Omega}^{\mu}\right) \psi=0
$$

where $\tilde{\Omega}_{\mu} \equiv \frac{i}{2} \tilde{\omega}_{\mu}^{a b} \tilde{\Sigma}_{a b}, \tilde{\Sigma}_{a b}$ and $\tilde{\omega}_{\mu}^{a b}$ are the Lorentz spinor generator and spin connection in the rainbow metric, respectively, and $\left\{\tilde{\gamma}_{\mu}, \tilde{\gamma}_{\nu}\right\}=2 \tilde{g}_{\mu \nu}(E)$. To obtain the Hamilton-Jacobi equation for the fermion, the ansatz for $\psi$ is assumed as

$$
\psi=\exp \left(\frac{i I}{\hbar}\right) v
$$


where $v$ is a slowly varying spinor amplitude. Substituting eqn. (111) into eqn. (10), we find to the lowest order of $\hbar$

$$
\tilde{\gamma}_{\mu} \partial^{\mu} I v=0
$$

Multiplying both sides of eqn. (12) from the left by $\tilde{\gamma}_{\nu} \partial^{\nu} I$, one gets

$$
\left[\tilde{g}_{\mu \nu}(E) \partial^{\mu} I \partial^{\nu} I\right] v=0
$$

Since $v$ is nonzero, the Hamilton-Jacobi equation for a massless fermionic particle is also given by eqn. (9).

The Maxwell's equations for a massless vector field $A_{\mu}$ is

$$
\tilde{\nabla}^{\mu} \tilde{F}_{\mu \nu}=0
$$

where $\tilde{F}_{\mu \nu}=\tilde{\nabla}_{\mu} A_{\nu}-\tilde{\nabla}_{\nu} A_{\mu}$. We then make the WKB ansatz

$$
A_{\mu}=a_{\mu} \exp \left(\frac{i I}{\hbar}\right)
$$

where $a_{\mu}$ is the polarization vector and $I$ is the action. Plugging the WKB ansatz into eqn. (14), we find that leading order of $\hbar$ gives

$$
\tilde{g}^{\nu \sigma}(E)\left(a_{\nu} \partial_{\sigma} I \partial_{\mu} I-a_{\mu} \partial_{\sigma} I \partial_{\nu} I\right)=0
$$

To simplify eqn. (16), one could impose the Lorentz gauge in the curved spacetime

$$
\tilde{\nabla}^{\mu} A_{\mu}=0
$$

where the leading order is

$$
\tilde{g}^{\nu \sigma}(E) a_{\nu} \partial_{\sigma} I=0
$$

By plugging eqn. (18) into eqn. (16), it shows that the Hamilton-Jacobi equation for a massless vector boson is also given by eqn. (9).

From eqn. (9), one finds that the Hamilton-Jacobi equation for a massless particle in the rainbow metric (6) becomes

$$
f^{2}\left(E / m_{p}\right) \frac{\left(\partial_{t} I\right)^{2}}{B(r)}=g^{2}\left(E / m_{p}\right)\left[B(r)\left(\partial_{r} I\right)^{2}+\frac{h^{a b}(x) \partial_{a} I \partial_{b} I}{C\left(r^{2}\right)}\right]
$$

To solve the Hamilton-Jacobi equation for the action $I$, we can employ the following ansatz

$$
I=-E t+W(r)+\Theta(x),
$$


where $E$ is the particle's energy. Plugging the ansatz into eqn. (19), we have differential equations for $W(r)$ and $\Theta(x)$

$$
\begin{gathered}
h^{a b}(x) \partial_{a} \Theta(x) \partial_{b} \Theta(x)=\lambda \\
\partial_{r} W_{ \pm}(r) \equiv p_{r}^{ \pm}=\frac{ \pm 1}{B(r)} \sqrt{\frac{E^{2} f^{2}\left(E / m_{p}\right)}{g^{2}\left(E / m_{p}\right)}-\lambda \frac{B(r)}{C\left(r^{2}\right)}}
\end{gathered}
$$

where $+/-$ denotes the outgoing/ingoing solutions and $\lambda$ is a constant. Using the residue theory for the semi circle, we get

$$
\operatorname{Im} W_{ \pm}(r)=\frac{ \pm \pi}{2 \kappa} \frac{E f\left(E / m_{p}\right)}{g\left(E / m_{p}\right)}
$$

where $\kappa=\frac{B^{\prime}\left(r_{h}\right)}{2}$. As shown in [40], the probability of a particle tunneling from inside to outside the horizon is

$$
P_{\text {emit }} \propto \exp \left[-\frac{2}{\hbar}\left(\operatorname{Im} W_{+}-\operatorname{Im} W_{-}\right)\right] .
$$

There is a Boltzmann factor in $P_{\text {emit }}$ with an effective temperature, which is

$$
T_{\text {eff }}=T_{0} \frac{g\left(E / m_{p}\right)}{f\left(E / m_{p}\right)},
$$

where we define $T_{0}=\frac{\hbar \kappa}{2 \pi}$ and take $k_{B}=1$.

\section{THERMODYNAMICS OF BLACK HOLES IN RAINBOW GRAVITY}

We now use the Heisenberg uncertainty principle to estimate the black hole's temperature. For a massless particle, the modified dispersion relation (11) becomes

$$
\frac{x f(x)}{g(x)}=y
$$

where $x=E / m_{p} \geq 0$ and $y=p / m_{p} \geq 0$. To obtain the black hole's temperature from eqn. (24), eqn. (25) is needed to be solved for $x$ in terms of $y$. In fact, one could have for $x$

$$
x=y h(y)
$$

where eqn. (25) is inverted to get the function $h(y)$ and $\lim _{y \rightarrow 0} h(y)=1$. The Heisenberg uncertainty principle gives a relation between the momentum $p$ of an emitted particle and the event horizon radius $r_{h}$ of the black hole[41, 42]

$$
y=p / m_{p} \sim \delta p / m_{p} \sim \hbar / m_{p} \delta x \sim m_{p} / r_{h}
$$


Substituting eqn. (27) into eqn. (24), we have for the black hole's temperature

$$
T=T_{0} \frac{E}{p}=T_{0} h\left(\frac{m_{p}}{r_{h}}\right) .
$$

The range of the left-hand side(LHS) of eqn. (25) determines the ranges of the values of $r_{h}$. Specifically, the maximum value of the LHS of eqn. (25), which is denoted by $y_{c r}$, gives that $r_{h} \geq \frac{m_{p}}{y_{c r}}$. If $y_{c r}$ is finite, it always predicts the existence of the black hole's remnant. For some functions $f(x)$ and $g(x)$, the domain of the LHS of eqn. (25) might be $\left[0, x_{c r}\right] /\left[0, x_{c r}\right)$ with $x_{c r}$ being finite rather than $[0, \infty)$. Thus, it gives that the energy of the particle $E \leq m_{p} x_{c r}$. If the domain is $[0, \infty)$, we simply set $x_{c r}=\infty$.

For the AC dispersion relation given in eqn. (3), eqn. (25) becomes

$$
\frac{x}{\sqrt{1-\eta x^{n}}}=y \text {. }
$$

If $\eta>0$, we find that $y_{c r}=0$. However, there is an upper bound $x_{c r}=\eta^{-1 / n}$ on $x$ to make the LHS of eqn. (29) real. If $\eta<0$, we have $x_{c r}=\infty$ and $y_{c r}=\infty$ for $0<n<2$ and $x_{c r}=\infty$ and $y_{c r}=(-\eta)^{-1 / 2}$ for $n=2$. For the case with $\eta<0$ and $n>2$, the LHS of eqn. (29) has a global maximum value $y_{0}$ at $x_{0}$, where we define

$$
\begin{aligned}
& x_{0}=\left(\frac{2-n}{2} \eta\right)^{-1 / n}, \\
& y_{0}=\sqrt{\frac{n-2}{n}}\left(\frac{2-n}{2} \eta\right)^{-1 / n} .
\end{aligned}
$$

Thus, it would appear that $y \leq y_{0}$ and $x<\infty$ since $x$ can go to infinity. In FIG. 1, we plot an example with $\eta=-1$ and $n=4$. If one solves eqn. (29) for $y<y_{0}$ in terms of $x$, there are always two solutions $x_{S}$ and $x_{L}$, where $x_{S}<x_{0}<x_{L}$. However, only the solution $x_{S}$ is Taylor expandable in $\eta$. The solution $x_{L}$ is a "runaways" solution since it does not exist in the limit of $\eta \rightarrow 0$. In [43], it has been argued that "runaways" solutions are not physical and hence should be discarded. A similar argument was also given in the framework of the Generalized Uncertainty Principle in [44]. Therefore, we will discard "runaways" solutions and keep only the solution $x_{S}$ in this paper. In this case, we have $x_{c r}=x_{0}$ instead of

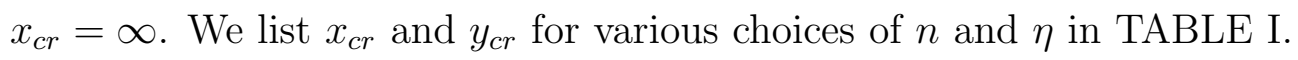

To calculate the black hole's temperature from eqn. (28), we need to solve eqn. (29) for 
$h(y)$. Nevertheless, it is non-trivial to get $h(y)$ for a general $n$. For $n=1,2,4$, we have

$$
h(y)=\left\{\begin{array}{rl}
\frac{1}{2}\left(-\eta y+\sqrt{4+\eta^{2} y^{2}}\right) & n=1 \\
\left(1+\eta y^{2}\right)^{-1 / 2} & n=2 . \\
y^{-2} \sqrt{\frac{\sqrt{1+4 \eta y^{4}}-1}{2 \eta}} & n=3
\end{array}\right.
$$

If $y \ll 1$, one has $x \ll 1$ and hence eqn. (29) becomes

$$
y=x\left(1+\frac{\eta x^{n}}{2}+\mathcal{O}\left(x^{2 n}\right)\right),
$$

which gives

$$
h(y)=1-\frac{\eta y^{n}}{2}+\mathcal{O}\left(y^{2 n}\right) .
$$

Since $x=E / m_{p}$ and $y=p / m_{p}$, the AC dispersion relation for a massless particle (29) leads to the group velocity of the particle

$$
\frac{1}{v_{g}}=\frac{\partial y}{\partial x}=\frac{1}{\sqrt{1-\eta x^{n}}}+\frac{n \eta x^{n}}{2\left(1-\eta x^{n}\right)^{3 / 2}} .
$$

If $\eta>0$, eqn. (34) gives $v_{g}<1$, which means the particle is subluminal. If $\eta<0, v_{g}>1$ and hence the particle is superluminal.

We now calculate the temperatures and entropies of a Schwarzschild black hole and a static uncharged black string with negative cosmological constant.

\begin{tabular}{|c|c|c|c|c|c|c|c|}
\hline & $x_{c r}$ & $y_{c r}$ & $M_{c r}^{S C}$ & $T_{c r}^{S C} / m_{p}$ & $M_{c r}^{B S}$ & $T_{c r}^{B S} / m_{p}$ & Lines in figures \\
\hline$\eta=0$ & $\infty$ & $\infty$ & 0 & $\infty$ & 0 & 0 & Blue Solid \\
\hline$\eta>0$ & $\eta^{-1 / n}$ & $\infty$ & 0 & $\frac{\eta^{-1 / n}}{4 \pi}$ & 0 & 0 & Black Solid \\
\hline$\eta<0,0<n<1$ & $\infty$ & $\infty$ & 0 & $\infty$ & 0 & $\infty$ & \\
\hline$\eta<0, n=1$ & $\infty$ & $\infty$ & 0 & $\infty$ & 0 & $-\eta \frac{3 \alpha^{2} m_{p}^{2}}{4 \pi}$ & Black Dashed \\
\hline$\eta<0,1<n<2$ & $\infty$ & $\infty$ & 0 & $\infty$ & 0 & 0 & \\
\hline$\eta<0, n=2$ & $\infty$ & $(-\eta)^{-1 / 2}$ & $\frac{m_{p}}{2(-\eta)^{-1 / 2}}$ & $\infty$ & $\frac{\alpha^{3} m_{p}^{3}}{4(-\eta)^{-3 / 2}}$ & $\infty$ & Red Dashed \\
\hline$\eta<0, n>2$ & $x_{0}$ & $y_{0}$ & $\frac{m_{p}}{2 y_{0}}$ & $\frac{x_{0}}{4 \pi}$ & $\frac{\alpha^{3} m_{p}^{3}}{4 y_{0}^{3}}$ & $\frac{3 \alpha^{2} m_{p}^{2}}{4 \pi} \frac{x_{0}}{y_{0}^{2}}$ & Red Solid \\
\hline
\end{tabular}

TABLE I: The values of $x_{c r}, y_{c r}, M_{c r}^{S C}, T_{c r}^{S C} / m_{p}, M_{c r}^{B S}$, and $T_{c r}^{B S} / m_{p}$ for various values of $(n, \eta)$. The superscripts $S C$ and $B S$ stand for the Schwarzschild black hole and black string, respectively. 


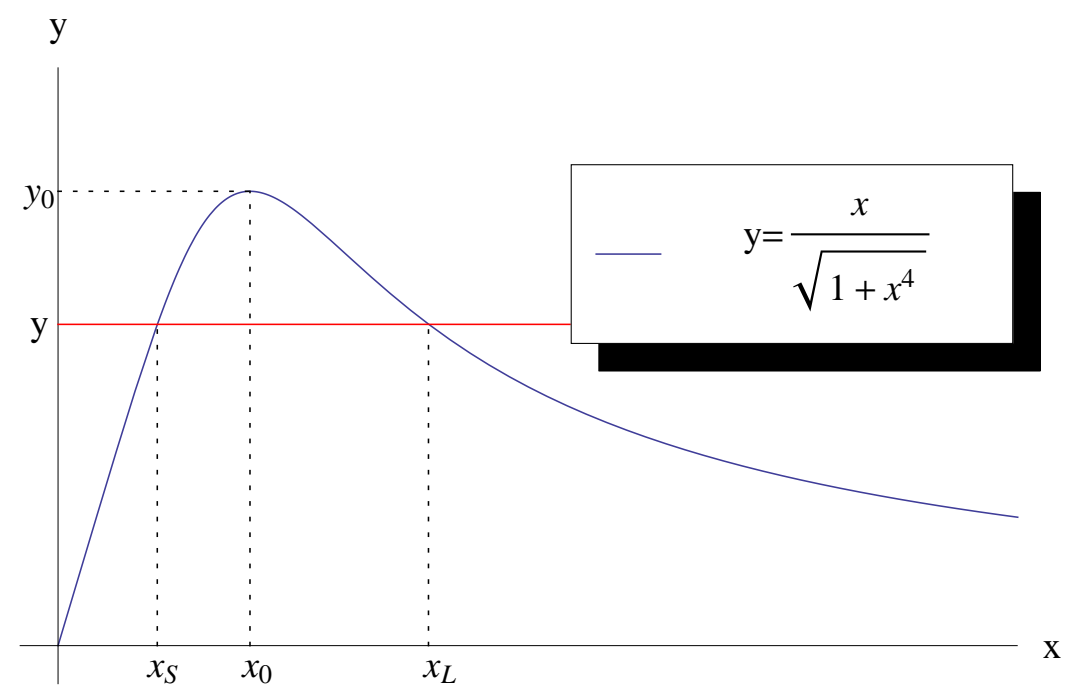

FIG. 1: Plot of the curve of $\frac{x}{\sqrt{1-\eta x^{n}}}$ with $\eta=-1$ and $n=4$. The curve has a maximum value $y_{0}=\frac{1}{\sqrt{2}}$ at $x_{0}=1$. For $y<y_{0}$, there are $x_{S}$ and $x_{L}$ with $x_{S}<x_{L}$ satisfying $\frac{x_{L / S}}{\sqrt{1+x_{L / S}^{4}}}=y$. The solution $x_{L}$ is considered runaways since it does not exist in the limit of $\eta \rightarrow 0$. Thus, only the solution $x_{S}$ is considered in the paper.

\section{A. Schwarzschild Black Hole}

For a Schwarzschild black hole with mass $M$, one has $B(r)=1-\frac{2 M}{r}$ and $r_{h}=2 M$. Thus, the temperature is

$$
T^{S C}=\frac{m_{p}^{2}}{8 \pi M} h\left(\frac{m_{p}}{2 M}\right) .
$$

If $M \gg m_{p}$, we have from eqn. (33) that

$$
T^{S C}=\frac{m_{p}^{2}}{8 \pi M}\left[1-\frac{\eta}{2^{n+1}} \frac{m_{p}^{n}}{M^{n}}+\mathcal{O}\left(\frac{m_{p}^{2 n}}{M^{2 n}}\right)\right] .
$$

The minimum mass $M_{c r}^{S C}$ of the black hole is given by

$$
M_{c r}^{S C}=\frac{m_{p}}{2 y_{c r}}
$$

When the mass $M$ reaches $M_{c r}^{S C}$, the temperature of the black hole is denoted by $T_{c r}^{S C}$. Eqn. (38) gives that

$$
T_{c r}^{S C}=\frac{x_{c r} m_{p}}{4 \pi}
$$

For $\eta<0$ and $n \geq 2, y_{c r}$ is finite and hence the black hole should have non-vanishing minimum mass $M_{c r}^{S C}$. This implies an existence of black hole remnant due to rainbow 


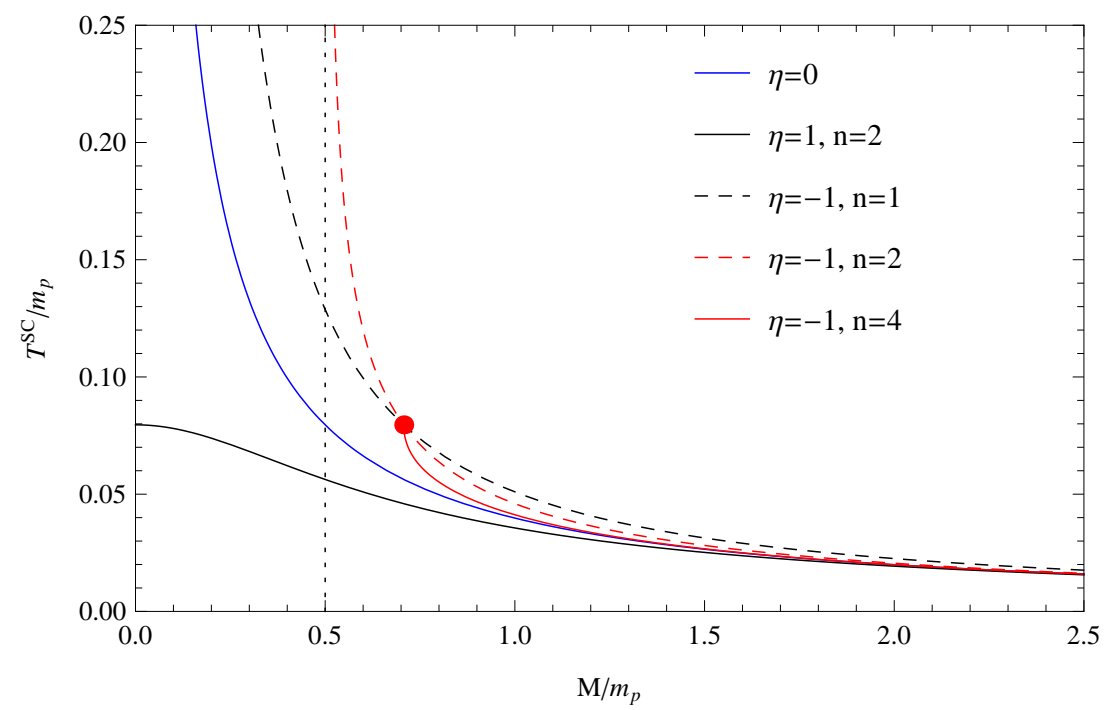

FIG. 2: Plot of the temperature $T^{S C} / m_{p}$ against the mass $M / m_{p}$ for a Schwarzschild black hole. All the lines asymptotically approach $T^{S C}=0$ as $M / m_{p} \rightarrow \infty$. The blue line is usual case, where $T^{S C}$ blows up as $M \rightarrow 0$. The red dot is the end of the red solid line, where the black hole has a remnant $M_{c r}^{S C}=1 / \sqrt{2} m_{p}$. In this case, $T^{S C}$ does not blow up as $M \rightarrow M_{c r}^{S C}$. The black dotted line is the asymptotic line of the red dashed line as $M \rightarrow M_{c r}^{S C}=0.5 m_{p}$, which is the black hole's remnant. In this case, $T^{S C}$ blows up as $M \rightarrow M_{c r}^{S C}$.

gravity. By eqn. (38), we find that $T_{c r}^{S C}$ for $n=2$ is infinite while $T_{c r}^{S C}$ for $n>2$ is $\frac{x_{0} m_{p}}{4 \pi}$, which is finite. For $\eta<0$ and $0<n<2$, we find that $M_{c r}^{S C}=0$ and $T_{c r}^{S C}=\infty$. In this case, the black hole would evaporate completely while its temperature increases and finally becomes infinity during evaporation, just like the standard Hawking radiation. For $\eta>0$, the black hole would also evaporate completely. However, the temperature of the black hole is a finite value $\frac{\eta^{-1 / n} m_{p}}{4 \pi}$ at the end of the evaporation process. We list $M_{c r}^{S C}$ and $T_{c r}^{S C}$ for all the possible values of $\eta$ and $n$ in TABLE I. In FIG. 2, we plot the temperature $T^{S C} / m_{p}$ against the black hole mass $M / m_{p}$ (both in Planck units), for examples with $(\eta, n)=(1,2)$, $(\eta, n)=(-1,1),(\eta, n)=(-1,2)$, and $(\eta, n)=(-1,4)$. The standard Hawking radiation is also plotted as a blue line in FIG. 2.

From the first law of black hole thermodynamics $d S=d M / T^{S C}$, the entropy associated with the temperature (35) can be given by

$$
S=\int_{M_{c r}^{S C}}^{M} \frac{d M}{T^{S C}}=2 \pi \int_{\frac{m_{p}}{2 M}}^{y_{c r}} \frac{d y}{y^{3} h(y)},
$$




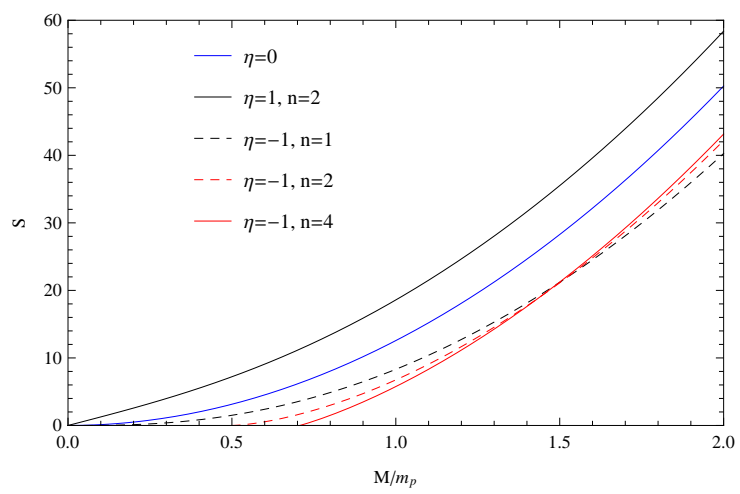

FIG. 3: Plot of the entropy $S$ against the mass $M / m_{p}$ for a Schwarzschild black hole.

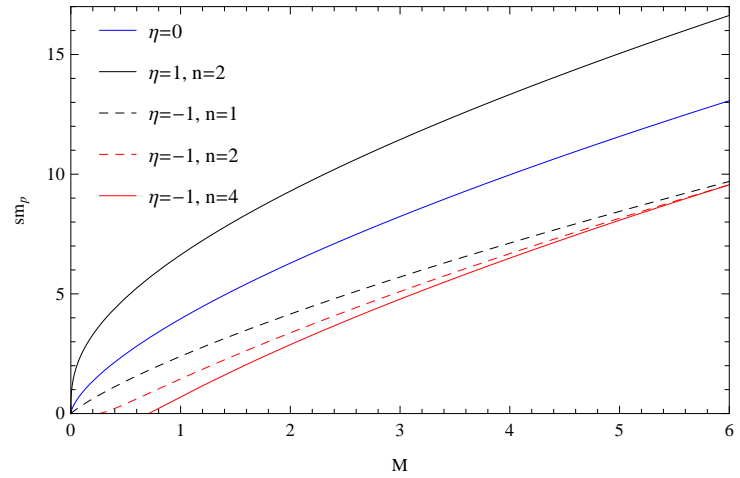

FIG. 4: Plot of the entropy of a Plank length $s m_{p}$ against the mass per unit length $M$ for a static uncharged black string.

where we use $y_{c r}=\frac{m_{p}}{2 M_{c r}^{S C}}$. For $\eta=0$, we have $h(y)=1$ and $y_{c r}=\infty$. Thus, eqn. (39) gives the Bekenstein-Hawking entropy

$$
S=\frac{4 \pi M^{2}}{m_{p}^{2}}=\frac{A}{4 \hbar} .
$$

where $A=4 \pi r_{h}^{2}$ is the area of the black hole. If $M \gg m_{p}(A \gg \hbar)$, eqn. (399) gives the entropy up to the subleading term

$$
S \sim\left\{\begin{array}{cr}
\frac{A}{4 \hbar}+\frac{\pi \eta}{2-n}\left(\frac{A}{4 \pi \hbar}\right)^{\frac{2-n}{2}} & 0<n<2 \\
\frac{A}{4 \hbar}+\frac{\pi \eta}{2} \ln \frac{A}{4 \pi \hbar} & n=2 \\
\frac{A}{4 \hbar}+S_{0} & n>2
\end{array}\right.
$$

where $S_{0}$ is a constant and we use eqn. (33). The leading terms of eqn. (41) are the familiar Bekenstein-Hawking entropy. For $n=2$, we obtain the logarithmic subleading term. In FIG. 3, we plot the entropy $S$ against the black hole mass $M / m_{p}$, for examples with $\eta=0$, $(\eta, n)=(1,2),(\eta, n)=(-1,1),(\eta, n)=(-1,2)$, and $(\eta, n)=(-1,4)$.

In [34], the temperature and entropy of a rainbow Schwarzschild black hole has also been computed in the case with $\eta>0$ and $n=2$. The temperature and logarithmic subleading term of the entropy obtained in our paper are the same as those in [34]. By contrast, the temperature and entropy were calculated in the case with $\eta>0$ in [33], where it was found that the black hole had a non-vanishing minimum mass and the temperature was zero at the minimum mass. As pointed out in [34], the difference between the results in our paper and [34] and those in [33] stems from the fact the MDR was partially used in [33]. In fact, 
the author in [33] used the MDR to get $T_{\text {eff }}$ while the ordinary dispersion relation rather than the MDR was used to obtain the relation between the energy $E$ of an emitted particle and the event horizon radius $r_{h}$.

\section{B. Static Uncharged Black String}

We consider the Einstein-Hilbert action in four dimensions with a negative cosmological constant $\Lambda$, which is given by

$$
S=\frac{1}{16 \pi} \int d^{4} x \sqrt{-g}(R-2 \Lambda),
$$

where $g$ is the determinant of the metric and $R$ the Ricci scalar. If we assume that the solution of the Einstein equations is cylindrically symmetric and time-independent, the line element for a static uncharged black string becomes[45]

$$
d s^{2}=\left(\alpha^{2} r^{2}-\frac{b}{\alpha r}\right) d t^{2}-\left(\alpha^{2} r^{2}-\frac{b}{\alpha r}\right)^{-1} d r^{2}-r^{2}\left(d \theta^{2}+\alpha^{2} d z^{2}\right)
$$

where

$$
\begin{gathered}
\alpha^{2}=-\frac{\Lambda}{3}, b=4 M \\
-\infty<t<\infty, 0 \leq r<\infty, \quad-\infty<z<\infty, 0 \leq \theta \leq 2 \pi .
\end{gathered}
$$

Here, $M$ is the mass per unit length of the $z$ line of black string.

For the static uncharged black string, we have $B(r)=\alpha^{2} r^{2}-\frac{b}{\alpha r}, r_{h}=b^{1 / 3} / \alpha$, and $\kappa=\frac{3 \alpha}{2} b^{1 / 3}$. Thus, eqn. (28) leads to the black string temperature

$$
\frac{T^{B S}}{m_{p}}=\frac{3 \alpha m_{p} b^{1 / 3}}{4 \pi} h\left(\frac{\alpha m_{p}}{b^{1 / 3}}\right) .
$$

If $b^{1 / 3} \gg \alpha m_{p}\left(M \gg \alpha^{3} m_{p}^{3}\right)$, we have for the temperature

$$
T^{B S}=\frac{3 \alpha m_{p} b^{1 / 3}}{4 \pi}\left[1-\frac{\eta}{2^{n+1}} \frac{\alpha^{n} m_{p}^{n}}{b^{n / 3}}+\mathcal{O}\left(\frac{\alpha^{2 n} m_{p}^{2 n}}{b^{2 n / 3}}\right)\right] .
$$

If $n>1$, one has

$$
T^{B S} \rightarrow T_{0} \text { as } M \rightarrow \infty
$$

where $T_{0}=\frac{3 \alpha m_{p} b^{1 / 3}}{4 \pi}$ is the black string temperature in the usual case. Since $r_{h} \geq \frac{m_{p}}{y_{c r}}$, the minimum mass per unit length $M_{c r}^{B S}$ of the black string is given by

$$
M_{c r}^{B S}=\frac{\alpha^{3} m_{p}^{3}}{4 y_{c r}^{3}}
$$




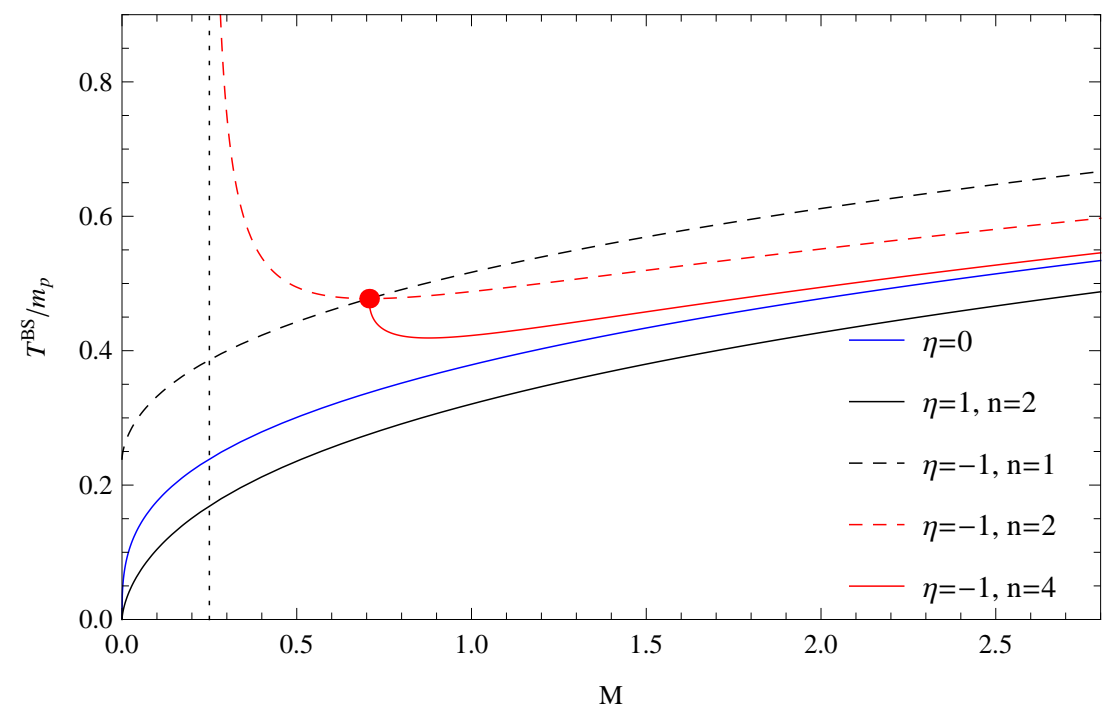

FIG. 5: Plot of the temperature $T^{B S} / m_{p}$ against the mass per unit length $M$ for a static uncharged black string. The lines with $n>1$ asymptotically approach the blue line as $M \rightarrow \infty$. The blue line is the usual case, where $T^{S C} \rightarrow 0$ as $M \rightarrow 0$. The red dot is the end of the red solid line, where the black string has a remnant $M_{c r}^{B S}=1 / \sqrt{2}$. The black dotted line is the asymptotic line of the red dashed line as $M \rightarrow M_{c r}^{B S}=0.25$, which is the black string's remnant. In this case, $T^{B S}$ blows up as $M \rightarrow M_{c r}^{B S}$.

When $M$ reaches $M_{c r}^{S C}$, the temperature of the black string becomes

$$
\frac{T_{c r}^{B S}}{m_{p}}=\frac{3 \alpha^{2} m_{p}^{2}}{4 \pi} \frac{x_{c r}}{y_{c r}^{2}} .
$$

For the usual case with $\eta=0$, we have $h(y)=1$ and $y_{c r}=\infty$. The minimum mass per unit length $M_{c r}^{B S}=0$ and $T^{B S}$ goes to zero as $M \rightarrow 0$. For $\eta<0$ and $n \geq 2$, $y_{c r}$ is finite and hence the black string should have non-vanishing minimum mass per unit length $M_{c r}^{B S}$, which indicates an existence of black string remnant. Using eqn. (48), we can show that $T_{c r}^{B S}$ for $n=2$ is infinity while $T_{c r}^{B S}$ for $n>2$ is finite. For $\eta>0$, the black string would evaporate completely with $T^{B S}$ goes to zero as $M \rightarrow 0$, which is the same as in the usual case. For $\eta<0$ and $0<n<2$, the minimum mass per unit length $M_{c r}^{B S}$ is zero. Nevertheless, we find that $T_{c r}^{B S}=\infty$ for $0<n<1, T_{c r}^{B S}=-\eta \frac{3 \alpha^{2} m_{p}^{3}}{4 \pi}$ for $n=1$, and $T_{c r}^{B S}=0$ for 0 . We list $M_{c r}^{B S}$ and $T_{c r}^{B S}$ for various values of $\eta$ and $n$ in TABLE I. In FIG. 5 where we set $\alpha m_{p}=1$, we plot the temperature $T^{B S} / m_{p}$ against the mass per unit length $M$, for examples with $\eta=0,(\eta, n)=(1,2),(\eta, n)=(-1,1),(\eta, n)=(-1,2)$, and $(\eta, n)=(-1,4)$.

Using the first law of black hole thermodynamics, we have for the entropy per unit length 
associated with the temperature (45)

$$
s=\int_{M_{c r}^{S C}}^{M} \frac{d M}{T^{S C}}=\frac{\pi \alpha m_{p}}{m_{p}} \int_{\frac{\alpha m_{p}}{(4 M)^{1 / 3}}}^{y_{c r}} \frac{d y}{y^{3} h(y)} .
$$

If $M \gg 1$, eqn. (49) gives the entropy per unit length up to the subleading term

$$
s \sim\left\{\begin{array}{cc}
\frac{\pi \alpha}{2} \frac{b^{2 / 3}}{\alpha^{2} m_{p}^{2}}+\frac{\alpha \pi \eta}{2(2-n)} \frac{b^{(2-n) / 3}}{\left(\alpha m_{p}\right)^{2-n}} & 0<n<2 \\
\frac{\pi \alpha}{2} \frac{b^{2 / 3}}{\alpha^{2} m_{p}^{2}}+\frac{\pi \alpha \eta}{2} \ln \frac{b^{1 / 3}}{\alpha m_{p}} & n=2 \\
\frac{\pi \alpha}{2} \frac{b^{2 / 3}}{\alpha^{2} m_{p}^{2}}+s_{0} & n>2
\end{array}\right.
$$

where $s_{0}$ is a constant. In FIG. 3, we plot $m_{p} S$ against $M$, for examples with $\eta=0$, $(\eta, n)=(1,2),(\eta, n)=(-1,1),(\eta, n)=(-1,2)$, and $(\eta, n)=(-1,4)$.

\section{LUMINOSITIES OF BLACK HOLES IN RAINBOW GRAVITY}

For particles emitted in a wave mode labelled by energy $E$ and quantum numbers $i$, we find that

(Probability for a black hole to emit a particle in this mode)

$=\exp \left(-\frac{E}{T_{e f f}}\right) \times($ Probability for a black hole to absorb a particle in the same mode),

where $T_{\text {eff }}$ is given by eqn. (24). The above relation for the usual dispersion relation was obtained by Hartle and Hawking [46] using semiclassical analysis. Neglecting back-reaction, detailed balance condition requires that the ratio of the probability of having $N$ particles in a particular mode to the probability of having $N-1$ particles in the same mode is

$\exp \left(-\frac{E}{T_{e f f}}\right)$. One then follows the argument in [40] to get the average number $n_{E, i}$ in the mode with $E$ and $i$

$$
n_{E, i}=n\left(\frac{E}{T_{e f f}}\right)
$$

where we define

$$
n(x)=\frac{1}{\exp x-(-1)^{\epsilon}} .
$$

Note that $\epsilon=0$ for bosons and $\epsilon=1$ for fermions.

As discussed in section $\amalg$, there is an upper bound $m_{p} x_{c r}$ on the energy $E$ of the particle. Moreover, another upper bound comes from the requirement that nothing can be emitted that lowers the energy below the remnant mass of a black hole. Thus, one has $E \leq M-M_{c r}$, 
where $M$ is the mass of the black hole and $M_{c r}$ is the remnant mass. Considering both upper bounds, we have for $E$

$$
E \leq E_{\max } \equiv \min \left\{m_{p} x_{c r}, M-M_{c r}\right\} .
$$

In [40, 47, 48], we have found that the total luminosity in the MDR case is given by

$$
L=\sum_{i} \int\left|T_{i}(E)\right|^{2} \omega n_{E, i} \frac{d E}{2 \pi \hbar} .
$$

where $E$ is the energy of the particle, $i$ are quantum numbers needed to specify a mode besides $E$, and $\left|T_{i}(E)\right|^{2}$ is the greybody factor. Usually, $T_{i}(E)$ represents the transmission coefficient of the black hole barrier which in general can depend on $E$ and $i$. The relevant radiations usually have the energy of order $\hbar M^{-1}$ for a black hole with the mass $M$ and one hence needs to use the wave equations to compute $T_{i}(E)$. However, solving the wave equations for $T_{i}(E)$ could be complex. On the other hand, we can use the geometric optics approximation to estimate $\left|T_{i}(E)\right|^{2}$. In the geometric optics approximation, we assume $E \gg M$ and high energy waves will be absorbed unless they are aimed away from the black hole. Hence $\left|T_{i}(E)\right|^{2}=1$ for all the classically allowed $E$ and $i$, while $\left|T_{i}(E)\right|^{2}=0$ otherwise. For the usual dispersion relation, the well-known Stefan's law for black holes is obtained in this approximation.

In the remaining of the section, we will use the geometric optics approximation to calculate luminosities of a 2D rainbow black hole, a 4D rainbow spherically symmetric one, and a $4 \mathrm{D}$ rainbow cylindrically symmetric one. For simplicity, we assume that the particles are massless.

\section{A. 2D Black Hole}

Suppose the metric of a $2 \mathrm{D}$ black hole is given by

$$
d s^{2}=B(r) d t^{2}-\frac{1}{B(r)} d r^{2},
$$

where $B(r)$ has a simple zero at $r=r_{h}$. In this case, we have $|T(E)|^{2}=1$ when $E \leq E_{\max }$ and $|T(E)|^{2}=0$ otherwise. By eqn. (154), the luminosity of a $2 \mathrm{D}$ black hole is

$$
L=g_{s} \int_{0}^{E_{\max }} E n\left(\frac{E}{T_{0}} \frac{f\left(E / m_{p}\right)}{g\left(E / m_{p}\right)}\right) \frac{d E}{2 \pi \hbar},
$$


where we use eqn. (24) for $T_{\text {eff }}$ and $T_{0}=\frac{m_{p}^{2} \kappa}{2 \pi}$ with $\kappa=\frac{B^{\prime}\left(r_{h}\right)}{2}$. Note $g_{s}$ is the number of polarization which is 1 for scalars and 2 for spin- $1 / 2$ fermion and vector bosons. Defining

$$
u=\frac{E}{T_{0}} \frac{f\left(E / m_{p}\right)}{g\left(E / m_{p}\right)},
$$

we find

$$
E=T_{0} u h\left(\frac{u T_{0}}{m_{p}}\right) .
$$

Using eqn. (57) to change variables in eqn. (56), we have for the luminosity

$$
L=\frac{g_{s} T_{0}^{2}}{2 \pi m_{p}^{2}} \int_{0}^{u_{\max }} h\left(\frac{u T_{0}}{m_{p}}\right)\left[h\left(\frac{u T_{0}}{m_{p}}\right)+\left(\frac{u T_{0}}{m_{p}}\right) h^{\prime}\left(\frac{u T_{0}}{m_{p}}\right)\right] \text { un }(u) d u,
$$

where we define

$$
u_{\max }=\frac{E_{\max }}{T_{0}} \frac{f\left(\frac{E_{\max }}{m_{p}}\right)}{g\left(\frac{E_{\max }}{m_{p}}\right)} .
$$

Note that the change of variables given in eqn. (57) is legitimate for the integral (59) if the function $\frac{x f(x)}{g(x)}$ is monotonic over $\left(0, x_{c r}\right)$, which is the case for the AC dispersion relation.

Now investigate properties of the luminosity for the AC dispersion relation given in eqn. (3). If $T_{0} \ll m_{p}$, eqn. (159) becomes

$$
L \approx \frac{g_{s} T_{0}^{2}}{2 \pi m_{p}^{2}} \int_{0}^{\infty}\left[1-\frac{n+2}{2} \eta\left(\frac{u T_{0}}{m_{p}}\right)^{n}\right] \text { un }(u) d u
$$

where we set $u_{\max }=\infty$. For the emission of $n_{s}$ species of massless particles of spin $s$, we have

$$
\begin{aligned}
L & \approx \frac{\pi}{12}\left(\frac{T_{0}}{m_{p}}\right)^{2}\left\{\left(n_{0}+2 n_{1}+n_{1 / 2}\right)\right. \\
& \left.-\frac{3(n+1) !(n+2)}{\pi^{2}}\left(\frac{T_{0}}{m_{p}}\right)^{n} \eta\left[L i_{n+2}(1)\left(n_{0}+2 n_{1}\right)+\frac{2-2^{-n}}{n+2} \zeta(n+2) n_{1 / 2}\right]\right\},
\end{aligned}
$$

where $L i_{s}(z)$ is the polylogarithm of order $s$ and argument $z$ and $\zeta(z)$ is the zeta function. If $\eta>0(\eta<0)$, the luminosity becomes smaller(larger) than that in the usual case with $\eta=0$. This is expected from eqn. (24) where $T_{\text {eff }}$ is lowered(raised) due to rainbow gravity if $\eta>0(\eta<0)$.

During the late stage of the black hole evaporation process when $\frac{M-M_{c r}}{m_{p}} \ll \min \left\{1, x_{c r}\right\}$, one has $\frac{E_{\max }}{m_{p}}=\frac{M-M_{c r}}{m_{p}} \ll 1$ and hence

$$
u_{\max } \approx \frac{m_{p}}{T_{0}} \frac{M-M_{c r}}{m_{p}}\left[1+\frac{\eta}{2}\left(\frac{M-M_{c r}}{m_{p}}\right)^{n}\right]
$$


The luminosity of radiation of one species of bosons is

$$
L=\frac{g_{s} T_{0}}{2 \pi m_{p}} \frac{M-M_{c r}}{m_{p}}\left[1-\frac{\eta}{2} \frac{1}{n+1}\left(\frac{M-M_{c r}}{m_{p}}\right)^{n}+\mathcal{O}\left(\left(\frac{M-M_{c r}}{m_{p}}\right)^{2 n}\right)\right],
$$

and that of radiation of one species of fermions is

$$
L=\frac{g_{s}}{4 \pi}\left(\frac{M-M_{c r}}{m_{p}}\right)^{2}\left[1+\mathcal{O}\left(\left(\frac{M-M_{c r}}{m_{p}}\right)^{2 n}\right)\right]
$$

where $g_{s}$ is the number of polarization. From eqns. (64) and (65), we find that the black hole evaporates mostly via bosons in the late stage of the black hole evaporation process.

\section{B. 4D Spherically Symmetric Black Hole}

For a 4D spherically symmetric black hole with $h_{a b}(x) d x^{a} d x^{b}=d \theta^{2}+\sin ^{2} \theta d \phi^{2}$ in eqn. (5), we have found $\lambda$ in eqn. (21) is given by [40]

$$
\lambda=\left(l+\frac{1}{2}\right)^{2} \hbar^{2}
$$

where $l=0,1, \cdots$ is the angular momentum. Since $p_{r}$ in eqn. (21) is always a real number in the geometric optics approximation, one has an upper bound on $\lambda$

$$
\lambda \leq \frac{C\left(r^{2}\right)}{B(r)} \frac{f^{2}\left(E / m_{p}\right)}{g^{2}\left(E / m_{p}\right)} E^{2} .
$$

Suppose $\frac{C\left(r^{2}\right)}{B(r)}$ has a minimum at $r_{\text {min }}$. If the particles overcome the angular momentum barrier and get absorbed by the black hole, one has

$$
\lambda \leq \lambda_{\max } \equiv \frac{C\left(r_{\min }^{2}\right)}{B\left(r_{\min }\right)} \frac{f^{2}\left(E / m_{p}\right)}{g^{2}\left(E / m_{p}\right)} E^{2} .
$$

The luminosity is

$$
\begin{aligned}
L & =g_{s} \sum_{l}(2 l+1) \int E n_{E, l} \frac{d E}{2 \pi \hbar} \\
& =g_{s} \int_{0}^{E_{\max }} \frac{E d E}{2 \pi \hbar^{3}} n\left(\frac{E}{T_{0}} \frac{f\left(E / m_{p}\right)}{g\left(E / m_{p}\right)}\right) \int_{0}^{\lambda_{\max }} d\left[\left(l+\frac{1}{2}\right)^{2}\right] \\
& =\frac{g_{s}}{2 \pi \hbar^{3}} \frac{C\left(r_{\min }^{2}\right)}{B\left(r_{\min }\right)} \int_{0}^{E_{\max }} n\left(\frac{E}{T_{0}} \frac{f\left(E / m_{p}\right)}{g\left(E / m_{p}\right)}\right) \frac{f^{2}\left(E / m_{p}\right)}{g^{2}\left(E / m_{p}\right)} E^{3} d E,
\end{aligned}
$$


where we use eqn. (51) for $n_{E, l}$. Making change of variables for eqn. (69)

$$
u=\frac{E}{T_{0}} \frac{f\left(E / m_{p}\right)}{g\left(E / m_{p}\right)}
$$

the luminosity of a $4 \mathrm{D}$ spherically symmetric black hole becomes

$$
L=\frac{g_{s} T_{0}^{4}}{2 \pi m_{p}^{4}} \frac{C\left(r_{\min }^{2}\right)}{B\left(r_{\min }\right) m_{p}^{2}} \int_{0}^{u_{\max }} h\left(\frac{u T_{0}}{m_{p}}\right)\left[h\left(\frac{u T_{0}}{m_{p}}\right)+\left(\frac{u T_{0}}{m_{p}}\right) h^{\prime}\left(\frac{u T_{0}}{m_{p}}\right)\right] u^{3} n(u) d u .
$$

In what follows, we focus on the $\mathrm{AC}$ dispersion relation. If $T_{0} \ll m_{p}$, eqn. (71) becomes

$$
L \approx \frac{g_{s} T_{0}^{4}}{2 \pi m_{p}^{4}} \frac{C\left(r_{\min }^{2}\right)}{B\left(r_{\min }\right) m_{p}^{2}} \int_{0}^{\infty}\left[1-\frac{n+2}{2} \eta\left(\frac{u T_{0}}{m_{p}}\right)^{n}\right] u^{3} n(u) d u,
$$

where we set $u_{\max }=\infty$. For the emission of $n_{s}$ species of massless particles of spin $s$, we have

$$
\begin{aligned}
L & \approx \frac{\pi^{3}}{30}\left(\frac{T_{0}}{m_{p}}\right)^{4} \frac{C\left(r_{\min }^{2}\right)}{m_{p}^{2} B\left(r_{\min }\right)}\left\{\left(n_{0}+2 n_{1}+\frac{7}{4} n_{1 / 2}\right)\right. \\
& \left.-\frac{15(n+3) !(n+2)}{2 \pi^{4}}\left(\frac{T_{0}}{m_{p}}\right)^{n} \eta\left[L i_{n+4}(1)\left(n_{0}+2 n_{1}\right)+\left(2-2^{-n-2}\right) \zeta(n+4) n_{1 / 2}\right]\right\},
\end{aligned}
$$

where $L i_{s}(z)$ is the polylogarithm of order $s$ and argument $z$ and $\zeta(z)$ is the zeta function. In the late stage of the black hole evaporation process with $\frac{M-M_{c r}}{m_{p}} \ll \min \left\{1, x_{c r}\right\}$, one finds that the luminosity of radiation of one species of bosons is

$$
L=\frac{g_{s} T_{0}}{6 \pi m_{p}} \frac{C\left(r_{\min }^{2}\right)}{m_{p}^{2} B\left(r_{\min }\right)}\left(\frac{M-M_{c r}}{m_{p}}\right)^{3}\left[1+\frac{3 \eta}{2(n+3)}\left(\frac{M-M_{c r}}{m_{p}}\right)^{n}+\mathcal{O}\left(\left(\frac{M-M_{c r}}{m_{p}}\right)^{2 n}\right)\right],
$$

and that of radiation of one species of fermions

$$
L=\frac{g_{s}}{8 \pi} \frac{C\left(r_{\min }^{2}\right)}{m_{p}^{2} B\left(r_{\min }\right)}\left(\frac{M-M_{c r}}{m_{p}}\right)^{4}\left[1+\frac{4 \eta}{n+4}\left(\frac{M-M_{c r}}{m_{p}}\right)^{n}+\mathcal{O}\left(\left(\frac{M-M_{c r}}{m_{p}}\right)^{2 n}\right)\right],
$$

where $g_{s}$ is the number of polarization. Similar to a $2 \mathrm{D}$ black hole, the evaporation via bosons dominates the $4 \mathrm{D}$ spherically symmetric black hole evaporation process in the late stage.

In the geometric optics approximation, a 4D spherically symmetric black hole can be described as a black sphere for absorbing particles. The total luminosity are determined 
by the radius of the black sphere $R$ and the temperature of the black hole $T$. For the AC dispersion relation, we have for massless particles

$$
R=\sqrt{\frac{\lambda_{\max }}{E^{2}}}=\frac{C\left(r_{\min }^{2}\right)}{B\left(r_{\min }\right)} \frac{1}{1-\eta\left(E / m_{p}\right)^{n}} \text { and } T=T_{e f f}=T_{0} \sqrt{1-\eta\left(E / m_{p}\right)^{n}} .
$$

If $\eta>0(\eta<0)$, the radius $R$ becomes larger(smaller) than that in the usual case while the effective temperature becomes lower(higher) due to rainbow gravity. For the subluminal case with $\eta>0$ (the superluminal case with $\eta<0$ ), the competition between the increased(decreased) radius and the decreased(increased) temperature determines whether the luminosity would increase or decrease. For $T_{0} \ll m_{p}$, it appears from eqn. (73) that the effects of decreased(increased) temperature wins the competition and hence the luminosity tends to become smaller(larger). However for the late stage of the evaporation process, it seems from eqns. (74) and (75) that the effects of increased(decreased) radius wins the competition and hence the luminosity becomes larger(smaller).

We now work with a Schwarzschild black hole to investigate more properties of the black hole's luminosity. For a Schwarzschild black hole, one has $B(r)=1-\frac{2 M}{r}, r_{h}=2 M$, $\kappa=\frac{1}{4 M}, T_{0}=\frac{m_{p}^{2}}{8 \pi M}, r_{\min }=3 M$ and $\frac{C\left(r_{\min }^{2}\right)}{B\left(r_{\min }\right)}=27 M^{2}$. For $M \gg m_{p}$, it shows form eqn. (73) that the corrections to the luminosity from rainbow gravity effects are around $\mathcal{O}\left(\frac{m_{p}}{M}\right)^{n}$. Nevertheless, these corrections begin to become appreciable around $M \lesssim M_{*} \equiv$ $\frac{c_{n}^{1 / n}}{8 \pi}|\eta|^{1 / n} m_{p}$ when the second term in the bracket of eqn. (73) becomes comparable to 1. Here $c_{n}$ is the numerical factor in front of $n_{s}$ of the second term in the bracket and $c_{n}^{1 / n} \sim 5-10$ for $1 \leq n \leq 10$. If $M_{c r}^{S C}=0$, in the late stage of the evaporation process with $\frac{M}{m_{p}} \ll \min \left\{1, x_{c r}\right\}$, the corrections to the luminosity are around $\mathcal{O}\left(\frac{M^{n}}{m_{p}^{n}}\right)$. Similarly, these corrections are important when $M \gtrsim M_{* *} \equiv|\eta|^{-1 / n} m_{p}$. Thus, we can conclude that for cases with $M_{c r}^{S C}=0$, the effects of rainbow gravity impacts the black hole's luminosity noticeably when $M_{* *} \lesssim M \lesssim M_{*}$. For cases with non-vanishing $M_{c r}^{S C}$, the the black hole's luminosity deviates from that in the usual case appreciably when $M \lesssim M_{*}$. In FIG. 6, we plot the luminosity $L$ of radiation of one species of bosons against $M / m_{p}$ for examples with $\eta=0,(\eta, n)=(1,2),(\eta, n)=(-1,1),(\eta, n)=(-1,2)$, and $(\eta, n)=(-1,4)$. Note that the effects of rainbow gravity does not change the black hole's luminosity appreciably in FIG. [6 since $M_{*} \sim|\eta|^{1 / n} m_{p}=|\eta|^{-1 / n} m_{p} \sim M_{* *}$ for $|\eta|=1$. Due to the requirement that the energy $E$ of emitted particles could not exceed $M-M_{c r}^{S C}$, the luminosities in all cases approach zero as $M \rightarrow M_{c r}^{S C}$. 


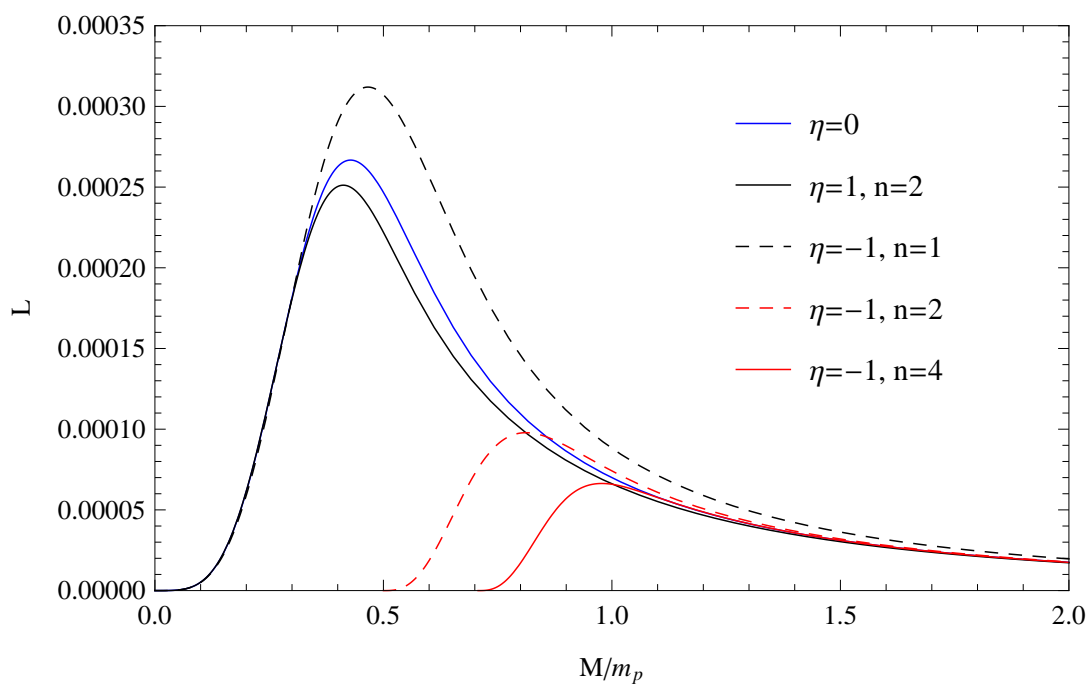

FIG. 6: Plot of the luminosity $L$ of radiation of one species of bosons against the mass $M / m_{p}$ for a Schwarzschild black hole. The blue line is the usual case. All the other lines asymptotically approach the blue line as $M \gg 1$. For the cases with the minimal mass $M_{c r}^{S C}=0$, the black lines asymptotically approach the blue line as $M \rightarrow 0$. For all the lines, the luminosities approach zero as $M \rightarrow M_{c r}^{S C}$.

To study the sensitivity of the $\mathrm{BH}$ dynamics to the parameter $\eta$, we plot the luminosity $L$ of radiation of one species of bosons against $M / m_{p}$ for different values of $\eta$ in FIG. 7. Since $M_{*} \sim|\eta|^{1 / n} m_{p}$ and $M_{* *} \sim|\eta|^{-1 / n} m_{p}$, we find that the larger $|\eta|$ is, the more apparent the effects of rainbow gravity on the luminosities become. In FIG. 7, we plot three cases as follows:

(a) Subluminal cases where $\eta>0$ and $M_{c r}^{S C}=0$. An example with $n=2$ is plotted in FIG. 7(a) for $\eta=1,10,100$, and 1000 . The red solid line $(\eta=1000)$ can be barely seen since it is too close to the horizontal axis. It is evident that the luminosity for $M_{* *} \lesssim M \lesssim M_{*}$ gets more suppressed as the parameter $\eta$ becomes larger. In other words, the effects of rainbow gravity could dramatically slow down the evaporation process of the black hole for large enough $\eta$. Hence, the corresponding characteristic time scale for the black hole to evaporate from $M_{*}$ to $M_{* *}$ could be much longer than in the usual case.

(b) Superluminal cases with the non-vanishing remnant mass $M_{c r}^{S C}$ where $\eta<0$ and $n \geq 2$. An example with $n=4$ is plotted in FIG. 7(b) for $\eta=-1,-10,-100$, and 


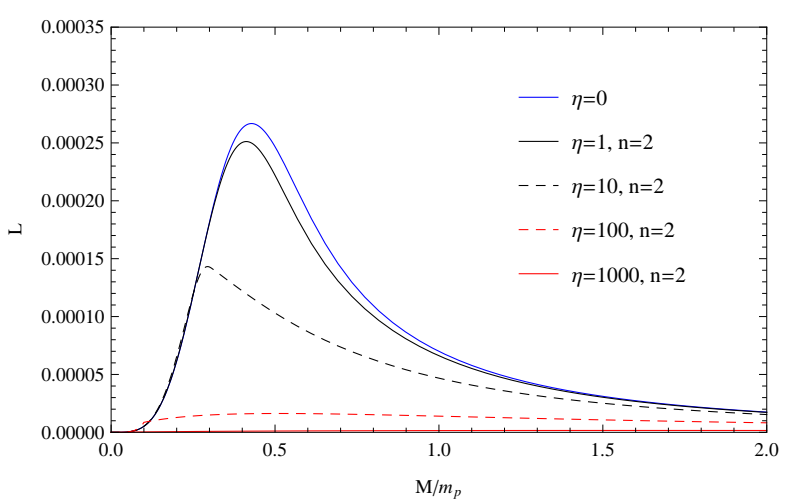

(a) Subluminal cases

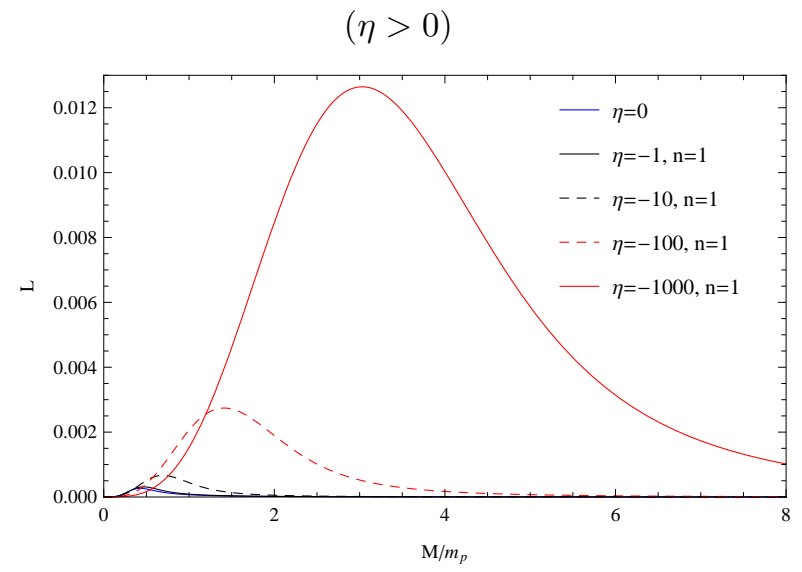

(c) Superluminal cases without remnant

$$
(\eta<0 \text { and } 0<n<2)
$$

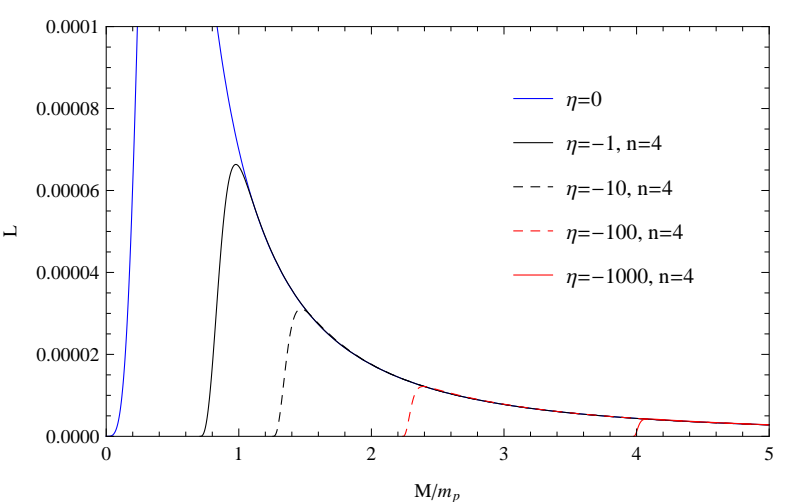

(b) Superluminal cases with remnant $(\eta<0$ and $n \geq 2)$

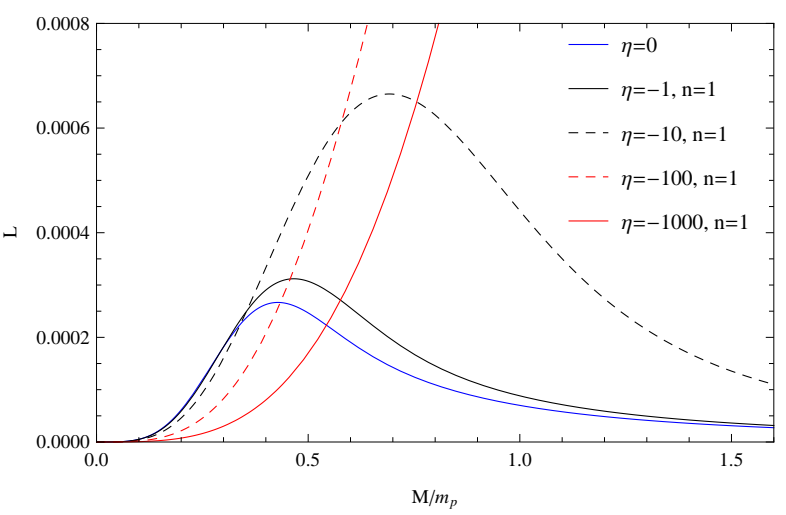

(d) Superluminal cases without remnant

$(\eta<0$ and $0<n<2)$

FIG. 7: Plots of the luminosity $L$ of radiation of one species of bosons against the mass $M / m_{p}$ for a Schwarzschild black hole. The luminosity $L$ is plotted in subluminal cases and superluminal cases with and without remnant for various values of $\eta$.

-1000. In this case, eqn. (37) gives that $M_{c r}^{S C}=\frac{m_{p}|\eta|^{1 / n}}{\sqrt{2}} \gtrsim M_{*}$. It shows that the luminosity starts to deviate from that in the usual case when $M$ is close to $M_{c r}^{S C}$ and then decreases to zero once $M=M_{c r}^{S C}$. Note that $M_{c r}^{S C}$ becomes larger as we increase $|\eta|$.

(c) Superluminal cases with $M_{c r}^{S C}=0$ where $\eta<0$ and $0<n<2$. An example with $n=1$ is plotted in FIGs. 7(c) and $7(\mathrm{~d})$ for $\eta=-1,-10,-100$, and -1000 . Opposite to the subluminal cases, the effects of rainbow gravity could dramatically boost the luminosity for $M_{* *} \lesssim M \lesssim M_{*}$ in this case if $|\eta|$ is large enough. For example, the 
maximum value of the luminosity for $\eta=-1000$ is approximately $10^{3}$ times greater than that in the usual case and $10^{20}$ times greater for $\eta=-10^{16}$, for which the energy scale of Lorentz-invariance violation is assumed around $1 \mathrm{TeV}$. The rainbow gravity speeds up the process of evaporation and hence the characteristic time scale for the black hole to evaporate from $M_{*}$ to $M_{* *}$ becomes shorter than in the usual case.

The luminosity for a Schwarzschild black hole has also been calculated in the framework of rainbow gravity in [32], where massless bosons were considered and the authors used the MDR of the form of

$$
E^{2}-p^{2}(1-\lambda p)=m^{2}
$$

By contrast, there are a number of differences between the calculations in our paper and in [32], which are as follows:

1. The geometric optics approximation have been used to calculate the luminosity of a black hole in both papers. In such approximation, a Schwarzschild black hole can be described as a black sphere of the radius $R$ and the temperature $T$. As a result, when the luminosity is calculated in the framework of rainbow gravity, we have considered effects of rainbow gravity on both $R$ and $T$. On the other hand, only effects of rainbow gravity on $T$ were considered in [32]. As discussed before, the corrections to $R$ could play an important role in the late stage of the evaporation process. This can be illustrated by FIG. 7(d), where the corrections to $R$ dominate over those to $T$ and make the luminosity smaller than in the usual case for small enough $M / m_{p}$, although the corrections to $T$ tend to increase the luminosity.

2. In our paper, we used detailed balance condition to show that the average number $n_{E, i}$ in the mode with $E$ and $i$ is

$$
n_{E, i}=n\left(\frac{E}{T_{e f f}}\right)
$$

where $T_{\text {eff }}$ is given in eqn. (24). In contrast, the authors of [32] assumed an average behavior for particles described by a unique average temperature, which is $T^{S C}$ obtained in section III. Therefore in [32], the average number $n_{E, i}$ was given by

$$
n_{E, i}=n\left(\frac{E}{T^{S C}}\right) \text {. }
$$


3. We have required the particles' energy $E \leq \min \left\{m_{p} x_{c r}, M-M_{c r}\right\}$ while only $E \leq$ $m_{p} x_{c r}$ was used in [32]. The extra requirement $E \leq M-M^{c r}$ could dramatically change behaviors of the evaporation process in the late stage. In fact, for the usual case with $\eta=0, x_{c r}=\infty$, and $M_{c r}=0$, one finds the luminosity of bosons without imposing $E \leq M-M^{c r}$ is $L \sim M^{-2}$, which implies that the black hole would evaporate completely in finite time and have a final explosion at $M=0$. However, if the requirement $E \leq M-M^{c r}$ is imposed, the luminosity $L \sim M^{5}$ for small enough $M$. Thus, it takes infinite time for the black hole to evaporate completely and death of the black hole is much milder. In our paper, since a black hole in rainbow gravity shares the similar the late-stage behaviors with that in the usual case, the lifetime of the rainbow black hole is also infinite. In contrast, the lifetime of a rainbow black hole in [32] turned out to be finite.

\section{4D Cylindrically Symmetric Black Hole}

For a 4D cylindrically symmetric black hole with $h_{a b}(x) d x^{a} d x^{b}=d \theta^{2}+\alpha^{2} d z^{2}$ in eqn. (5)), we have found that $\lambda$ in eqn. (21) is [40]

$$
\lambda=j^{2} \hbar^{2}+\frac{J_{z}^{2}}{\alpha^{2}},
$$

where $j$ is the angular momentum along $z$-axis and $J_{z}$ is a constant. To count the number of modes of radiation, we assume the length of the black string is $a$. Thus, the periodicity condition along $z$-axis gives

$$
J_{z}=\frac{2 \pi k \hbar}{a} \text { with } k \in Z .
$$

In the geometric optics approximation, eqn. (21) puts an upper bound on $\lambda$

$$
\lambda \leq \lambda_{\max } \equiv \frac{C\left(r_{\min }^{2}\right)}{B\left(r_{\min }\right)} \frac{f^{2}\left(E / m_{p}\right)}{g^{2}\left(E / m_{p}\right)} E^{2},
$$


where $\frac{C\left(r^{2}\right)}{B(r)}$ has a minimum at $r_{\text {min }}$. The luminosity per unit length $l$ is

$$
\begin{aligned}
l & \equiv \frac{L}{a}=\frac{g_{s}}{a} \sum_{j, k} \int E n\left(\frac{E}{T_{0}} \frac{f\left(E / m_{p}\right)}{g\left(E / m_{p}\right)}\right) \frac{d E}{2 \pi \hbar} \\
& =\frac{\alpha g_{s}}{4 \pi^{2} \hbar^{3}} \int d(j \hbar) d\left(\frac{J_{z}}{\alpha}\right) \int E n\left(\frac{E}{T_{0}} \frac{f\left(E / m_{p}\right)}{g\left(E / m_{p}\right)}\right) d E \\
& =\frac{\alpha g_{s}}{2 \pi \hbar^{3}} \frac{C\left(r_{\min }^{2}\right)}{B\left(r_{\min }\right)} \int_{0}^{E_{\max }} \frac{f^{2}\left(E / m_{p}\right)}{g^{2}\left(E / m_{p}\right)} E^{3} n\left(\frac{E}{T_{0}} \frac{f\left(E / m_{p}\right)}{g\left(E / m_{p}\right)}\right) d E \\
& =\frac{\alpha g_{s} T_{0}^{4}}{2 \pi m_{p}^{4}} \frac{C\left(r_{\min }^{2}\right)}{B\left(r_{\min }\right) m_{p}^{2}} \int_{0}^{u_{\max }} h\left(\frac{u T_{0}}{m_{p}}\right)\left[h\left(\frac{u T_{0}}{m_{p}}\right)+\left(\frac{u T_{0}}{m_{p}}\right) h^{\prime}\left(\frac{u T_{0}}{m_{p}}\right)\right] u^{3} n(u) d u
\end{aligned}
$$

where $u=\frac{E}{T_{0}} \frac{f\left(E / m_{p}\right)}{g\left(E / m_{p}\right)}$.

For a static uncharged black string (43), one has

$$
T_{0}=\frac{3 \alpha m_{p}}{4 \pi} b^{1 / 3}, r_{\min }=\infty, \text { and } \frac{C\left(r_{\min }^{2}\right)}{B\left(r_{\min }\right)}=\alpha^{-2},
$$

where $b=4 M$. Since the length of the black string is infinite, one only has $E \leq E_{\max } \equiv$ $m_{p} x_{c r}$. The luminosity per unit length for a static uncharged black string is

$$
\begin{aligned}
& l=\alpha \frac{81 m_{p}^{2} \alpha^{2} g_{s}}{512 \pi^{5}} b^{\frac{4}{3}} \int_{0}^{\frac{4 \pi y_{c r}}{3 \alpha m_{p} b^{1 / 3}}} h\left(\frac{3 \alpha m_{p}}{4 \pi} b^{1 / 3} u\right) \\
& {\left[h\left(\frac{3 \alpha m_{p}}{4 \pi} b^{1 / 3} u\right)+\left(\frac{3 \alpha m_{p}}{4 \pi} b^{1 / 3} u\right) h^{\prime}\left(\frac{3 \alpha m_{p}}{4 \pi} b^{1 / 3} u\right)\right] u^{3} n(u) d u .}
\end{aligned}
$$

In the cases with $M_{c r}^{B S}=0$, if $T_{0} \ll m_{p}(M \ll 1)$, for the emission of $n_{s}$ species of massless particles of spin $s$, we have

$$
\begin{aligned}
l & \approx \alpha \frac{81 m_{p}^{2} \alpha^{2}}{512 \pi^{5}} b^{\frac{4}{3}}\left\{\left(n_{0}+2 n_{1}+n_{1 / 2}\right)\right. \\
& \left.-\frac{3(n+1) !(n+2)}{\pi^{2}}\left(\frac{3 \alpha m_{p}}{4 \pi} b^{1 / 3} u\right)^{n} \eta\left[L i_{n+2}(1)\left(n_{0}+2 n_{1}\right)+\frac{2-2^{-n}}{n+2} \zeta(n+2) n_{1 / 2}\right]\right\},
\end{aligned}
$$

where $L i_{s}(z)$ is the polylogarithm of order $s$ and argument $z$ and $\zeta(z)$ is the zeta function. From eqn. (85), we find that $\frac{d M}{d t} \equiv l \sim M^{\frac{4}{3}}$ for $M \ll 1$. Consequently, just like the usual case, we have for the rainbow black string that $M \sim t^{-3}$, which means that its lifetime is infinite.

In the following, we will compute the asymptotic value of $l$ when $T_{0} \gg m_{p}(M \gg 1)$. In the usual case, the luminosity per unit length $l \propto M^{\frac{4}{3}}$. For other cases, the results show as follows: 
(a) Subluminal cases where $\eta>0$ and $M_{c r}^{B S}=0$. Using

$$
x=y h(y) \sim \eta^{-1 / n}-\frac{\eta^{-3 / n}}{n y^{2}},
$$

in eqn. (84), we have for the luminosity per unit length

$$
l \sim \frac{\eta^{-\frac{4}{n}} g_{s}}{\pi n m_{p}^{2} \alpha} \int_{\frac{4 \pi}{3 \alpha m_{p}(4 M)^{1 / 3}}}^{\infty} \frac{n(\tilde{u})}{\tilde{u}} d \tilde{u} .
$$

Thus, the luminosity per unit length of radiation of one species of bosons is

$$
l \sim \frac{3 \eta^{-\frac{4}{n}} g_{s}}{4 n \pi^{2} m_{p}}(4 M)^{1 / 3},
$$

and that of radiation of one species of fermions is

$$
l \sim \frac{\eta^{-\frac{4}{n}} g_{s}}{3 n \pi m_{p}^{2} \alpha} \ln M
$$

The luminosity per unit length $l$ is lower than that in the usual case for large $M$. In addition, the radiation of bosons dominates the evaporation process for $M \gg 1$. It is evident that $l$ becomes smaller as $\eta$ is increased. An example of one species of bosons with $\eta=1$ and $n=2$ is plotted as a black line in FIG. 8(a). Additionally, we plot $l$ against $M$ for the examples with $n=4$ in FIG. 8(b) for $\eta=1,10,100$, and 1000 .

(b) Superluminal cases with the non-vanishing remnant mass $M_{c r}^{B S}$ where $\eta<0$ and $n \geq 2$. In this case, one has for radiation of one species of bosons

$$
l \sim \frac{3 g_{s}}{8 \pi^{2}} \frac{(4 M)^{1 / 3}}{m_{p}} \int_{0}^{y_{c r}} h(y)\left[h(y)+y h^{\prime}(y)\right] y^{2} d y
$$

and radiation of one species of fermions

$$
l \sim \frac{g_{s}}{2 \pi \alpha m_{p}^{2}} \int_{0}^{y_{c r}} h(y)\left[h(y)+y h^{\prime}(y)\right] y^{3} d y .
$$

Similar to the subluminal cases, $l$ could become much lower than that in the usual case for large enough $M$ and the radiation of bosons dominates the evaporation process for $M \gg 1$. An example of one species of bosons with $\eta=-1$ and $n=4$ is plotted as a red solid line in FIG. 8(a).

(c) Superluminal cases with $M_{c r}^{B S}=0$ where $\eta<0$ and $0<n<2$. Using

$$
x=y h(y) \sim(-\eta)^{\frac{1}{2-n}} y^{\frac{2}{2-n}}
$$




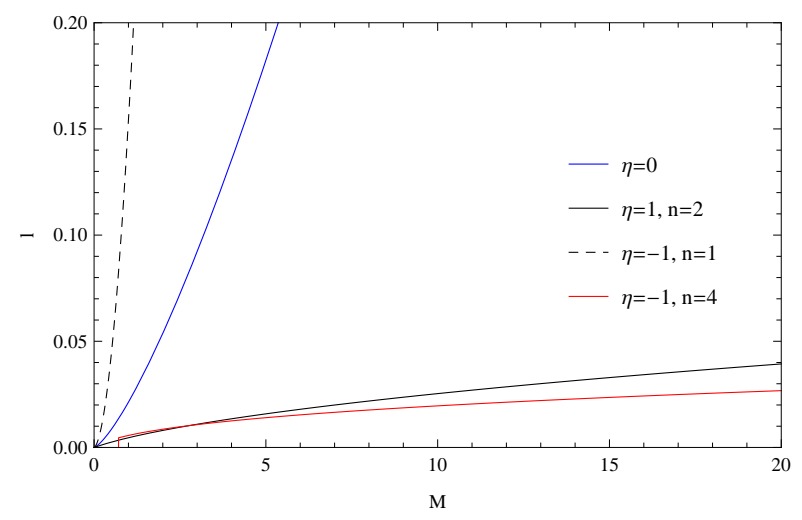

(a) Subluminal cases and superluminal cases with

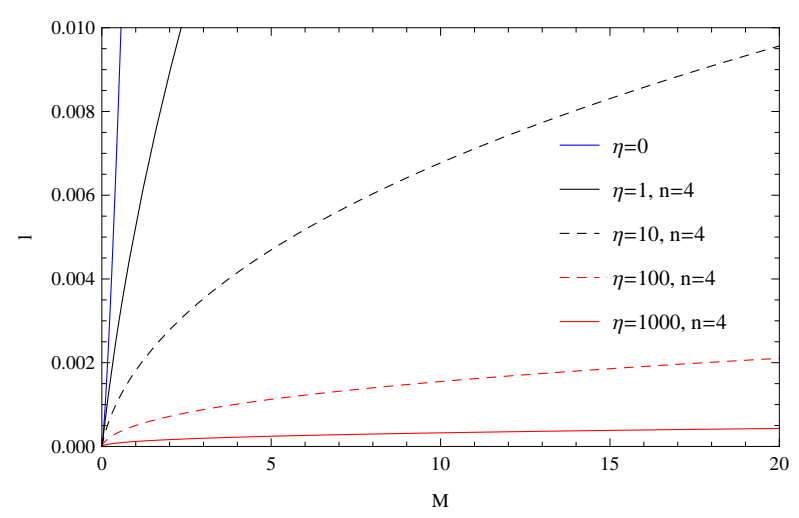

(b) Subluminal cases for various values of $\eta$

and without remnant

FIG. 8: Plots of the luminosity per unit length $l$ of radiation of one species of bosons against the mass per unit length $M$ for a static uncharged black string.

in eqn. (84), we find

$$
l \sim \frac{(-\eta)^{\frac{2}{2-n}} g_{s}}{2-n} \frac{81 m_{p}^{2} \alpha^{3}}{256 \pi^{5}}\left(\frac{3 \alpha m_{p}}{4 \pi}\right)^{\frac{n}{2-n}}(4 M)^{\frac{8-3 n}{3(2-n)}} \int_{0}^{\infty} u^{2} n(u) u^{\frac{2+n}{2-n}} d u
$$

Since $\frac{8-3 n}{3(2-n)}>\frac{4}{3}$ for $0<n<2, l$ could become much larger than that in the usual case for large enough $M$. The larger $|\eta|$ is, the faster the black string evaporates for $M \gg 1$. An example of one species of bosons with $\eta=-1$ and $n=1$ is plotted as a black dashed line in FIG. 8(a).

\section{CONCLUSION}

In this paper, we have analyzed the effects of rainbow gravity on the temperatures, entropies and luminosities of black holes. Using the Hamilton-Jacobi method for scalars, spin $1 / 2$ fermions and vector bosons, we first obtained the effective temperature $T_{\text {eff }}$ of a black hole, which depends on the energy $E$ of emitted particles. By relating the momentum $p$ of particles to the event horizon radius $r_{h}$ of the black hole, the temperatures of a rainbow Schwarzschild black hole and a rainbow static uncharged black string were calculated. Focusing on the AC dispersion relation with $f(x)=1$ and $g(x)=\sqrt{1-\eta x^{n}}$, we computed their minimum masses $M_{c r}$ and final temperatures $T_{c r}$ for different values of $\eta$ and $n$. All the results were listed in TABLE II In addition, a non-vanishing minimum mass indicates the 
existence of the black hole's remnant, which could shed light on the "information paradox". The entropies were also studied in section III.

In section IV, we used the geometric optics approximation to compute luminosities of a 2D black hole, a Schwarzschild one and a static uncharged black string in the framework of rainbow gravity. It was found that the luminosity of the rainbow Schwarzschild black hole with the mass $M$ deviates from that in the usual case only when $M_{* *} \lesssim M \lesssim M_{*}$ for $M_{c r}^{S C}=0$ or $M_{c r}^{S C} \leq M \lesssim M_{*}$ for a non-vanishing $M_{c r}^{S C}$, where $M_{*} \sim|\eta|^{1 / n} m_{p}$ and $M_{* *} \sim|\eta|^{-1 / n} m_{p}$. In the subluminal cases where $\eta>0$ and $M_{c r}^{S C}=0$, FIG. $7(\mathrm{a})$ shows that the effects of rainbow gravity could significantly suppress the luminosity for large $\eta$. In the superluminal cases with $M_{c r}^{S C}=0$ where $\eta<0$ and $0<n<2$, FIG. 7(c)] shows that the luminosity could be significantly boosted for large $|\eta|$. Similar results for the rainbow static uncharged black string with the mass per unit length $M \gg 1$ were also obtained for the subluminal cases and superluminal cases with $M_{c r}^{B S}=0$.

If the energy scale of Lorentz-invariance violation is $\Lambda$, the naturalness in effective field theories implies that $|\eta| \sim\left(\frac{\Lambda}{m_{p}}\right)^{n}$. For a Schwarzschild black hole, the effects of rainbow gravity start to play an important role when the mass of the black hole $M \lesssim|\eta|^{1 / n} m_{p} \sim \Lambda$. If Lorentz invariance is violated by quantum gravity, the natural scale $\Lambda \sim m_{p}$. Currently, there are no experimental evidence that Lorentz symmetry is violated in nature, which might suggest that $1 \mathrm{TeV} \lesssim \Lambda \lesssim m_{p}$. The experimental and observational constraints on Lorentz-invariance violation are reviewed in [49, 50]. As a result, the rainbow gravity plays a negligible role for stellar or galactic supermassive black holes. However, the possible production of $\mathrm{TeV}$-scale black holes at the LHC or ultra-high-energy cosmic ray collisions is predicted by low-scale quantum gravity[51]. Another possible source of small black holes is primordial black holes, which are created by primordial density fluctuations in the early universe and evaporate for enough long time. Future studies investigating the implications of our results on the rich phenomenology of these small black holes would be interesting.

\section{Acknowledgments}

We are grateful to Houwen $\mathrm{Wu}$ and Zheng Sun for useful discussions. This work is supported in part by NSFC (Grant No. 11005016, 11175039 and 11375121) and the Fundamental 
Research Funds for the Central Universities.

[1] S. W. Hawking, "Particle Creation by Black Holes," Commun. Math. Phys. 43, 199 (1975) [Erratum-ibid. 46, 206 (1976)].

[2] W. G. Unruh, "Notes on black hole evaporation," Phys. Rev. D 14, 870 (1976).

[3] G. Amelino-Camelia, "Testable scenario for relativity with minimum length," Phys. Lett. B 510, 255 (2001) hep-th/0012238].

[4] G. Amelino-Camelia, "Relativity in space-times with short distance structure governed by an observer independent (Planckian) length scale," Int. J. Mod. Phys. D 11, 35 (2002) gr-qc/0012051.

[5] J. Magueijo and L. Smolin, "Lorentz invariance with an invariant energy scale," Phys. Rev. Lett. 88, 190403 (2002) hep-th/0112090].

[6] J. Magueijo and L. Smolin, "Generalized Lorentz invariance with an invariant energy scale," Phys. Rev. D 67, 044017 (2003) gr-qc/0207085.

[7] G. Amelino-Camelia, J. R. Ellis, N. E. Mavromatos, D. V. Nanopoulos and S. Sarkar, "Tests of quantum gravity from observations of gamma-ray bursts," Nature 393, 763 (1998) astro-ph/9712103.

[8] D. Colladay and V. A. Kostelecky, "Lorentz violating extension of the standard model," Phys. Rev. D 58, 116002 (1998) hep-ph/9809521.

[9] S. R. Coleman and S. L. Glashow, "High-energy tests of Lorentz invariance," Phys. Rev. D 59, 116008 (1999) hep-ph/9812418.

[10] G. Amelino-Camelia and T. Piran, "Planck scale deformation of Lorentz symmetry as a solution to the UHECR and the TeV gamma paradoxes," Phys. Rev. D 64, 036005 (2001) astro-ph/0008107.

[11] T. Jacobson, S. Liberati and D. Mattingly, "TeV astrophysics constraints on Planck scale Lorentz violation," Phys. Rev. D 66, 081302 (2002) hep-ph/0112207].

[12] T. A. Jacobson, S. Liberati, D. Mattingly and F. W. Stecker, "New limits on Planck scale Lorentz violation in QED," Phys. Rev. Lett. 93, 021101 (2004) astro-ph/0309681.

[13] G. Amelino-Camelia, M. Arzano and A. Procaccini, "Severe constraints on loop-quantumgravity energy-momentum dispersion relation from black-hole area-entropy law," Phys. Rev. 
D 70, 107501 (2004) gr-qc/0405084.

[14] Y. Ling, B. Hu and X. Li, "Modified dispersion relations and black hole physics," Phys. Rev. D 73, 087702 (2006) gr-qc/0512083.

[15] G. Amelino-Camelia, M. Arzano, Y. Ling and G. Mandanici, "Black-hole thermodynamics with modified dispersion relations and generalized uncertainty principles," Class. Quant. Grav. 23, 2585 (2006) gr-qc/0506110.

[16] K. Nozari and A. S. Sefiedgar, "Comparison of approaches to quantum correction of black hole thermodynamics," Phys. Lett. B 635 (2006) 156 [gr-qc/0601116].

[17] A. S. Sefiedgar, K. Nozari and H. R. Sepangi, "Modified dispersion relations in extra dimensions," Phys. Lett. B 696, 119 (2011) [arXiv:1012.1406 [gr-qc]].

[18] B. Majumder, "Black Hole Entropy and the Modified Uncertainty Principle: A heuristic analysis," Phys. Lett. B 703, 402 (2011) arXiv:1106.0715 [gr-qc]].

[19] G. Amelino-Camelia, J. R. Ellis, N. E. Mavromatos and D. V. Nanopoulos, Int. J. Mod. Phys. A 12, 607 (1997) hep-th/9605211.

[20] G. Amelino-Camelia, Living Rev. Rel. 16, 5 (2013) [arXiv:0806.0339 [gr-qc]].

[21] J. Magueijo and L. Smolin, "Gravity's rainbow," Class. Quant. Grav. 21, 1725 (2004) gr-qc/0305055.

[22] A. F. Ali, M. Faizal and M. M. Khalil, "Remnant for all Black Objects due to Gravity's Rainbow," Nucl. Phys. B 894, 341 (2015) arXiv:1410.5706 [hep-th]].

[23] Y. Ling, "Rainbow universe," JCAP 0708, 017 (2007) gr-qc/0609129.

[24] Y. Ling and Q. Wu, "The Big Bounce in Rainbow Universe," Phys. Lett. B 687, 103 (2010) arXiv:0811.2615 [gr-qc]].

[25] R. Garattini and M. Sakellariadou, "Does gravity's rainbow induce inflation without an inflaton?," Phys. Rev. D 90, no. 4, 043521 (2014) [arXiv:1212.4987] [gr-qc]].

[26] A. Awad, A. F. Ali and B. Majumder, "Nonsingular Rainbow Universes," JCAP 1310, 052 (2013) arXiv:1308.4343 [gr-qc]].

[27] Y. Ling, X. Li and H. b. Zhang, "Thermodynamics of modified black holes from gravity's rainbow," Mod. Phys. Lett. A 22, 2749 (2007) gr-qc/0512084.

[28] P. Galan and G. A. Mena Marugan, "Entropy and temperature of black holes in a gravity's rainbow," Phys. Rev. D 74, 044035 (2006) gr-qc/0608061.

[29] H. Li, Y. Ling and X. Han, "Modified (A)dS Schwarzschild black holes in Rainbow spacetime," 
Class. Quant. Grav. 26, 065004 (2009) arXiv:0809.4819 [gr-qc]].

[30] R. Garattini, "Modified Dispersion Relations and Black Hole Entropy," Phys. Lett. B 685, 329 (2010) [arXiv:0902.3927 [gr-qc]].

[31] G. Salesi and E. Di Grezia, "Black hole evaporation within a momentum-dependent metric," Phys. Rev. D 79, 104009 (2009) arXiv:0902.3763 [gr-qc]].

[32] S. Esposito and G. Salesi, "Black hole dynamical evolution in a Lorentz-violating spacetime," Phys. Rev. D 83, 084043 (2011) arXiv:1012.4180 [gr-qc]].

[33] A. F. Ali, "Black hole remnant from gravity's rainbow," Phys. Rev. D 89, no. 10, 104040 (2014) arXiv:1402.5320 [hep-th]].

[34] Y. Gim and W. Kim, "Thermodynamic phase transition in the rainbow Schwarzschild black hole," JCAP 1410, 003 (2014) arXiv:1406.6475 [gr-qc]].

[35] P. Kraus and F. Wilczek, "Selfinteraction correction to black hole radiance," Nucl. Phys. B 433, 403 (1995) gr-qc/9408003.

[36] P. Kraus and F. Wilczek, "Effect of selfinteraction on charged black hole radiance," Nucl. Phys. B 437, 231 (1995) hep-th/9411219.

[37] K. Srinivasan and T. Padmanabhan, "Particle production and complex path analysis," Phys. Rev. D 60, 024007 (1999) gr-qc/9812028].

[38] M. Angheben, M. Nadalini, L. Vanzo and S. Zerbini, "Hawking radiation as tunneling for extremal and rotating black holes," JHEP 0505, 014 (2005) [hep-th/0503081].

[39] R. Kerner and R. B. Mann, "Tunnelling, temperature and Taub-NUT black holes," Phys. Rev. D 73, 104010 (2006) gr-qc/0603019].

[40] P. Wang and H. Yang, "Black Hole Radiation with Modified Dispersion Relation in Tunneling Paradigm: Static Frame," arXiv:1505.03045 [gr-qc].

[41] J. D. Bekenstein, "Black holes and entropy," Phys. Rev. D 7, 2333 (1973).

[42] R. J. Adler, P. Chen and D. I. Santiago, "The Generalized uncertainty principle and black hole remnants," Gen. Rel. Grav. 33, 2101 (2001) gr-qc/0106080.

[43] J. Z. Simon, "Higher Derivative Lagrangians, Nonlocality, Problems and Solutions," Phys. Rev. D 41, 3720 (1990).

[44] C. L. Ching and R. Parwani, "Scattering and Bound States of a Deformed Quantum Mechanics," arXiv:1207.1519 [hep-th].

[45] J. P. S. Lemos, "Cylindrical black hole in general relativity," Phys. Lett. B 353, 46 (1995) 
gr-qc/9404041].

[46] J. B. Hartle and S. W. Hawking, "Path Integral Derivation of Black Hole Radiance," Phys. Rev. D 13, 2188 (1976).

[47] B. Mu, P. Wang and H. Yang, "Minimal Length Effects on Tunnelling from Spherically Symmetric Black Holes," Adv. High Energy Phys. 2015, 898916 (2015) [arXiv:1501.06025 [gr-qc]].

[48] P. Wang, H. Yang and S. Ying, "Black Hole Radiation with Modified Dispersion Relation in Tunneling Paradigm: Free-fall Frame," arXiv:1505.04568 [gr-qc].

[49] D. Mattingly, "Modern tests of Lorentz invariance," Living Rev. Rel. 8, 5 (2005) gr-qc/0502097.

[50] S. Liberati, "Tests of Lorentz invariance: a 2013 update," Class. Quant. Grav. 30, 133001 (2013) arXiv:1304.5795 [gr-qc]].

[51] G. L. Landsberg, "Black holes at future colliders and beyond: A Review," hep-ph/0211043. 